Pesq. Vet. Bras. 31(5):459-463, maio 2011

\title{
Validação de um radioimunoensaio para a quantificação de metabólitos fecais de testosterona em hamster Sírio (Mesocricetus auratus) ${ }^{1}$
}

\author{
Marie-Odile M. Chelini2, Cláudio A. de Oliveira ${ }^{3}$ e Emma Otta ${ }^{2}$
}

\begin{abstract}
Chelini M.O.M., Oliveira C.A. \& Otta E. 2011. [Validation of a radioimmunoassay for the quantification of fecal testosterone metabolites in the Syrian hamster (Mesocricetus auratus).] Validação de um radioimunoensaio para a quantificação de metabólitos fecais de testosterona no hamster Sírio (Mesocricetus auratus). Pesquisa Veterinária Brasileira 31(5):459-463. Departamento de Psicologia Experimental, Instituto de Psicologia, Universidade de São Paulo, Av. Prof. Mello Moraes 1721, São Paulo, SP 05508-030, Brazil. E-mail: marodile@ usp.br

Besides being models for a variety of human diseases, hamsters have been object of numerous behavioral studies about stress and agonistic behavior. Such studies usually involve hormone change monitoring. The aim of the present study was to confirm the suitability of a commercial kit for human serum testosterone analysis to quantify testosterone metabolites in the feces (MFT) of male and female Syrian hamsters. Ten males were submitted to a GnRH challenge test to stimulate testicular activity and increase circulating testosterone levels. Five females received a testosterone injection and five other females were given a saline injection. Fecal samples collected before and after the procedures, and fecal samples collected from 20 pregnant females through gestation were analyzed for MFT with a commercial radioimmunoassay. The detection of a MFT peak $12 \mathrm{~h}$ after $\mathrm{GnRH}$ administration followed by a decrease under baseline showed that, in males, changes in the levels of MFT are related to respective changes of testosterone concentrations in the blood. Again in males a circadian cycle of MFT similar to the circadian rhythm described in literature for blood concentrations was detected. The administration of exogenous testosterone caused in females a dramatic peak of MFT. However, the concentrations measured through gestation did not reflect the pattern of endogenous testosterone blood levels as described in the literature. Therefore, the use of a radioimmunoassay for human blood testosterone was validated in male Syrian hamster, but a more specific assay would be necessary for female hamsters.
\end{abstract}

INDEX TERMS: Hamster, testosterone, fecal metabolites, non-invasive monitoring, endocrinology.

RESUMO.- Modelo para o estudo de diversas doenças humanas, o hamster tem sido objeto de numerosos estudos comportamentais e envolvendo estresse e/ou comportamento agonístico que supõem, muitas vezes, o monitoramento das flutuações hormonais nos indivíduos envolvidos. O objetivo do presente trabalho foi confirmar a adequação de um

\footnotetext{
${ }^{1}$ Recebido em 15 de julho de 2010.

Aceito para publicação em 8 de fevereiro de 2011.

2 Departamento de Psicologia Experimental, Instituto de Psicologia, Universidade de São Paulo (USP), Av. Prof. Mello Moraes 1721, São Paulo, SP 05508-030, Brasil. *Autor para correspondência: marodile@usp.br

${ }^{3}$ Departamento de Reprodução Animal, Faculdade de Medicina Veterinária e Zootecnia, USP, Av. Prof. Dr. Orlando Marques de Paiva 87, São Paulo, SP 05508270.
}

conjunto comercial para dosagem de testosterona em sangue humano para a quantificação de metabólitos fecais de testosterona (MFT) em hamsters Sírios machos e fêmeas. Dez machos foram submetidos a um desafio com um agonista de $\mathrm{GnRH}$ para estimular a atividade testicular, elevando os níveis circulantes de testosterona. Cinco fêmeas receberam uma injeção de testosterona enquanto cinco outras receberam uma injeção de solução salina. Amostras de fezes coletadas antes e depois dos procedimentos, assim como amostras fecais de 20 fêmeas gestantes coletadas ao longo da gestação foram analisadas com um conjunto comercial para radioimunoensaio. Um pico de MFT $12 \mathrm{~h}$ após a injeção seguido de uma queda abaixo do nível basal comprovou que, nos machos, as alterações nos níveis de MFT refletem as alterações da concentração de 
testosterona no sangue. Nestes observou-se um ciclo circadiano das concentrações de MFT com acrofase no início do período claro correspondendo ao ciclo descrito para as concentrações sanguíneas na literatura. Nas fêmeas a administração de testosterona exógena provocou uma elevação importante dos níveis de MFT, mas as concentrações medidas ao longo da gestação não refletiram o padrão dos níveis sanguíneos do hormônio endógeno. O conjunto para radioimunoensaio para testosterona em sangue humano mostrou-se adequado para o monitoramento da função testicular no hamster macho, mas um ensaio mais específico seria necessário para as fêmeas.

TERMOS DE INDEXAÇÃO: Hamster, testosterona, metabólitos fecais, monitoramento não invasivo, endocrinologia.

\section{INTRODUÇÃO}

A primeira ninhada de hamsters Sírios (Mesocricetus auratus) foi capturada em 1930. Desde então, estes roedores conquistaram um lugar de destaque entre as espécies utilizadas para a pesquisa médica e biológica graças a algumas características únicas que os tornam modelo para o estudo de diversas doenças humanas nas áreas de odontologia (Dale et al. 1944), cardiologia (Bajusz 1969, Missihoun et al. 2009), oncologia (Zheng et al. 2009) por exemplo. Ademais, espécie estritamente territorial, de hábitos solitários na natureza (Gattermann et al. 2001), o hamster tem sido objeto de numerosos estudos comportamentais e envolvendo estresse e/ou comportamento agonístico (Cain et al. 2004, Cordner et al. 2004, Taravosh-Lahn \& Delville 2004, Wommack et al. 2004, Gebhardt-Heinrich et al. 2007, Zhang et al. 2008). A investigação dos mecanismos fisiológicos subjacentes ao comportamento supõe muitas vezes o monitoramento das flutuações hormonais nos indivíduos envolvidos. Em se tratando de hormônios esteróides, métodos não invasivos como a quantificação de metabólitos fecais são cada vez mais usados em lugar das tradicionais dosagens sanguíneas, não somente para espécies selvagens de difícil manejo como também em animais de porte muito reduzido como os roedores de laboratório (Chelini et al. 2005, Chelini et al. 2006, Lepschy et al. 2007, Dias et al. 2008, Touma et al. 2009). Não exigindo nem anestesia, nem manipulação dos animais, estas técnicas não causam, per se, nenhum estresse adicional, podendo ser usadas em animais nos quais uma coleta de sangue poderia causar sérios danos, como fêmeas gestantes ou filhotes. Permitem a realização de estudos longitudinais envolvendo diversos hormônios que o volume de sangue exigido não permitiria levar a cabo pelos métodos convencionais de dosagem sanguínea e nos quais cada animal ou grupo é seu próprio controle. Obtém-se desta forma resultados mais acurados com um número menor de animais em respeito dos princípios humanitários que regem a experimentação animal. No entanto, como o metabolismo e a excreção dos esteróides não somente são mecanismos espécie-específicos, como ainda diferem às vezes entre os sexos dentro de uma mesma espécie (,Palme et al. 1996, Touma et al. 2003, Palme et al. 2005), tais técnicas devem ser validadas rigorosamente para cada espécie e cada sexo. $\mathrm{O}$ intuito desta validação é demonstrar que os ensaios utilizados têm a capacidade de detectar, nos níveis de metabólitos fecais dos esteróides, alterações que refletem as variações correspondentes, espontâneas ou provocadas, nas concentrações sanguíneas desses esteróides (Palme 2005, Dias et al. 2008).

Estudos anteriores já mostraram que é possível monitorar a função ovariana da fêmea de hamster através da quantificação dos metabólitos fecais de estradiol e de progesterona (Chelini et al. 2005), assim como a resposta adrenocortical ao estresse tanto de machos como de fêmeas analisando nas fezes os metabólitos de glicocorticóides (Chelini et al. 2010). Entretanto, não foi possível encontrar na literatura nenhuma validação, para o hamster, da dosagem de metabólitos fecais de testosterona. $\mathrm{O}$ objetivo do presente trabalho foi: 1) verificar se as variações diurnas das concentrações séricas de testosterona de hamsters machos se refletem nos níveis de metabólitos fecais de testosterona (MFT) 2) avaliar o efeito da administração de GnRH sobre as concentrações de MFT hamsters machos, 3) verificar o efeito da administração de testosterona exógena sobre as concentrações de MFT de hamsters fêmeas, 4) monitorar as concentrações de MFT de hamsters fêmeas ao longo da gestação, e 5) confirmar a adequação de um conjunto comercial para dosagem de testosterona em sangue humano para a quantificação de metabólitos de testosterona nas fezes de hamsters Sírios machos e fêmeas.

\section{MATERIAL E MÉTODOS}

Um total de 20 hamsters (10 machos e 10 fêmeas) adultos (100 dias de idade) foi adquirido do Biotério do Departamento de Patologia da Faculdade de Medicina Veterinária e Zootecnia da Universidade de São Paulo (FMVZ-USP). As salas deste biotério, onde os animais ficaram alojados até o fim do experimento, são submetidas a condições ambientais de 20 trocas de ar por hora, temperatura de $22 \pm 2^{\circ} \mathrm{C}$ e fotoperíodo de $12 \mathrm{~h}$ de claro por $12 \mathrm{~h}$ de escuridão com luzes acesas às 3h00. Ração para roedores de laboratório (Nuvilab CR1, Nuvital, Curitiba) e água filtrada foram fornecidas ad libitum. Os machos foram alojados individualmente e as fêmeas em dois grupos de cinco. Para facilitar a coleta de amostras fecais, a cama de maravalha foi substituída por papel absorvente substituído a cada coleta. As coletas noturnas foram realizadas sob luz vermelha fraca. Fezes contaminadas por urina não foram utilizadas. $O$ delineamento experimental obedeceu aos princípios éticos para o uso de animais de laboratório adotados pela Sociedade Brasileira de Ciência em Animais de Laboratório e foi aprovado pela Comissão de Bioética da FMVZ-USP (Certificado no 796/2005).

Os machos foram submetidos a um desafio com um agonista de $\mathrm{GnRH}$ para estimular a atividade testicular, elevando os níveis circulantes de testosterona. Cada animal recebeu uma injeção intramuscular de $0,1 \mathrm{ml}$ (equivalente a 2,5 $\mu \mathrm{g}$ ) de acetato de gonadorelina (Gestran Plus $₫$, Tecnopec Cons. Com. Repres. Ltda, São Paulo, Brasil). Amostras fecais individuais foram coletadas de 4 em 4 horas $24 \mathrm{~h}$ antes da injeção e $48 \mathrm{~h}$ depois. Nas fêmeas, uma injeção do mesmo produto teria estimulado a síntese de estradiol a partir da testosterona e não podíamos ter 
certeza de que, nem os níveis circulantes desta, nem as concentrações dos seus metabólitos fecais iriam elevar-se. Por esta razão, optamos por administrar às fêmeas o próprio hormônio, de modo a garantir a elevação de sua concentração no sangue e provavelmente nas fezes. Assim, cinco fêmeas receberam uma injeção intramuscular de $180 \mu \mathrm{L}$ (equivalente a $45 \mathrm{mg}$ ) de testosterona (Durateston®, Organon, São Paulo, Brasil). Cinco fêmeas formando o grupo controle receberam uma injeção intramuscular de $0,5 \mathrm{~mL}$ de solução salina isotônica estéril. A totalidade das fezes produzidas por cada grupo de fêmeas foi coletada diariamente durante quatro dias antes das injeções e quatro dias depois. Todas as injeções foram aplicadas às 12h, ou seja, três horas antes do apagar das luzes. Paralelamente, para confirmar a relevância fisiológica das medidas para os metabólitos de testosterona endógena, foi coletada diariamente, no fim do período claro, uma amostra fecal de cada uma de 20 fêmeas gestantes, durante seis dias antes do acasalamento e deste até o décimo quinto dia da gestação.

Os esteróides fecais foram extraídos por diluição em metanol segundo o protocolo recomendado por Palme (2005). As amostras foram pesadas e homogeneizadas com metanol $80 \%$ num multivortex, durante 20 minutos. A quantidade de metanol foi de $5 \mathrm{~mL}$ para as amostras pesando de $0,1 \mathrm{a} 0,5 \mathrm{~g}$, e de $1 \mathrm{~mL}$ para as amostras pesando menos de $0,1 \mathrm{~g}$. A seguir os tubos foram centrifugados durante 15 minutos a $500 \mathrm{x}$ g. O sobrenadante foi armazenado a $-20^{\circ} \mathrm{C}$ até a realização dos ensaios de dosagem.

As dosagens de MFT foram realizadas no Laboratório de Dosagens Hormonais do Departamento de Reprodução Animal da FMVZ-USP com conjuntos comerciais para radioimunoensaio (RIE) em fase sólida com tubos revestidos de anticorpo (Coat-A-Count testosterone $囚$, Siemens, Los Angeles, CA, EUA). Os parâmetros de controle de qualidade dos ensaios são a sensibilidade (menor concentração detectada) e os coeficientes de variação inter e intra-ensaio. Os coeficientes de variação foram todos inferiores a $10 \%$. O limite mínimo médio de detecção foi $1,80 \mathrm{ng} / \mathrm{dL}$. O anticorpo usado é altamente específico. As respectivas porcentagens de reação cruzada com outros esteróides são fornecidas pelo fabricante em [http://www.medicalsystems.it/ MetodicheSiemens/RIA/pitktt-5_siemens.pdf]. Todas as concentrações foram convertidas para ng/g de fezes (ng/g).

Após confirmação da normalidade da distribuição dos dados pelo teste de Kolmogorov-Smirnov e da homogeneidade das variâncias pelo teste de Levene, as concentrações médias dos diversos grupos nos diversos momentos foram comparadas entre si pelo teste $t$ para amostras independentes (com correção de Welch no caso de heterogeneidade de variâncias), ou pelo teste $t$ para amostras pareadas no caso de médias de um mesmo grupo. O nível de significância foi fixado em 0,05 para todas as comparações. Os resultados são fornecidos na forma de média e desvio padrão. Foram consideradas como valores de pico as concentrações superiores ao valor basal acrescentado de dois desvios padrões. Os resultados foram analisados com ajuda do software SPSS 13.0 para Windows.

\section{RESULTADOS}

Entre os machos as concentrações de MFT variaram numa faixa de 4,2 até 206,0ng/g antes do desafio com $\mathrm{GnRH}$, chegando a 445,0ng/g num animal em resposta a este. $O$ valor basal, calculado como a média das concentrações medidas nas fezes das 24 horas que precederam a injeção, foi de $65,0 \pm 48,6 \mathrm{ng} / \mathrm{g}$ com a média das concentrações me- didas 8 horas antes da injeção (ou seja, no início do período claro, $99,6 \pm 80,3 \mathrm{ng} / \mathrm{g}$ ) mais altas do que aquelas medidas $20 \mathrm{~h}$ antes dela (no início do período escuro, $48,1 \pm 26,4 \mathrm{ng} / \mathrm{g}$; teste $t$ para amostras pareadas $\mathrm{t}=2,700,8 \mathrm{gl}, \mathrm{p}=0,027$ ). Um pico de 175,8 129 ,2ng/g foi registrado 12 horas após a administração de $\mathrm{GnRH}$, seguido de um decréscimo das concentrações até um valor inferior ao nível basal $8 \mathrm{~h}$ mais tarde $(25,3 \pm 23,5 \mathrm{ng} / \mathrm{g}$; teste $t$ para amostras independentes com correção de Welch $t=2,326,12 d f, p=0,0384)$. No fim do experimento, $48 \mathrm{~h}$ após a injeção, as concentrações $(63,7 \pm 42,4 \mathrm{ng} / \mathrm{g})$ tinham voltado ao nível basal (Fig.1).

A concentração basal nas fêmeas, calculada como a média das concentrações medidas no grupo controle, foi de $15,5 \pm 7,4 \mathrm{ng} / \mathrm{g}$, valor aproximadamente quatro vezes inferior à concentração basal dos machos (teste tpara amostras independentes com correção de Welch, $\mathrm{t}=7,844,75 \mathrm{gl}$, $\mathrm{p}<0,0001$ ). No grupo experimental, a concentração basal foi determinada como média das concentrações medidas nos 5 dias que antecederam a administração de testosterona. Seu valor foi similar àquele do grupo controle $(18,1 \pm 10,6 \mathrm{ng} / \mathrm{g}$; test $t$ para amostras independentes

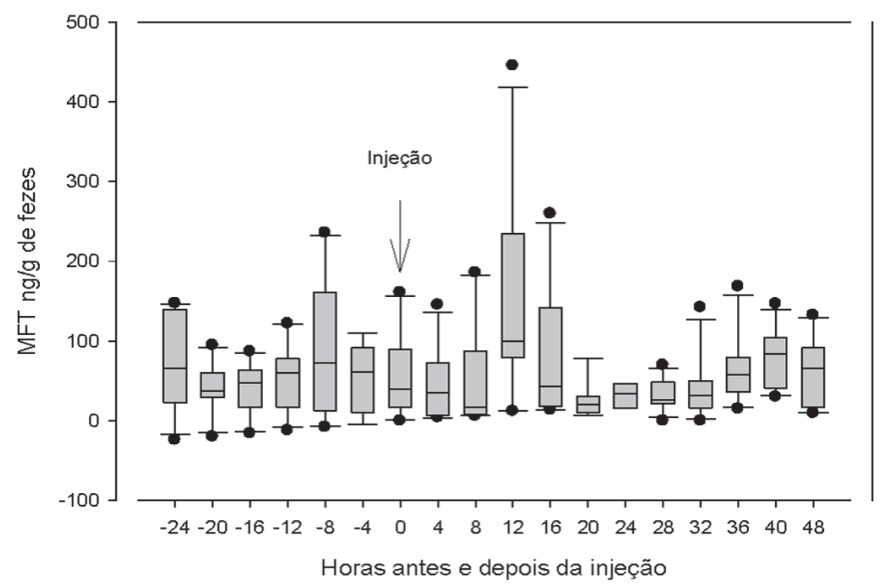

Fig.1. Concentrações de metabólitos fecais de testosterona (MFT) em 10 hamsters machos $24 \mathrm{~h}$ antes e $48 \mathrm{~h}$ depois de uma injeção de $\mathrm{GnRH}$.

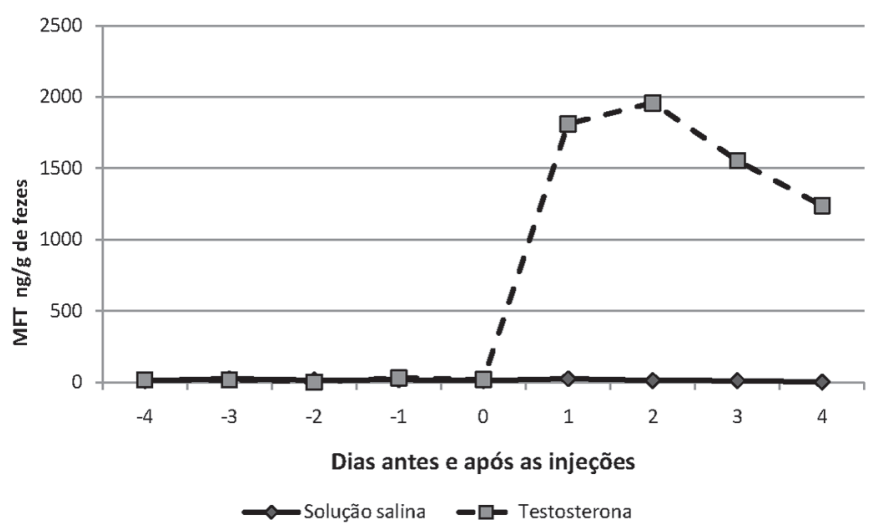

Fig.2. Variação da concentração de metabólitos fecais em dois grupos de hamsters fêmeas 4 dias antes e 4 dias depois de injeções de testosterona ou de solução salina. 


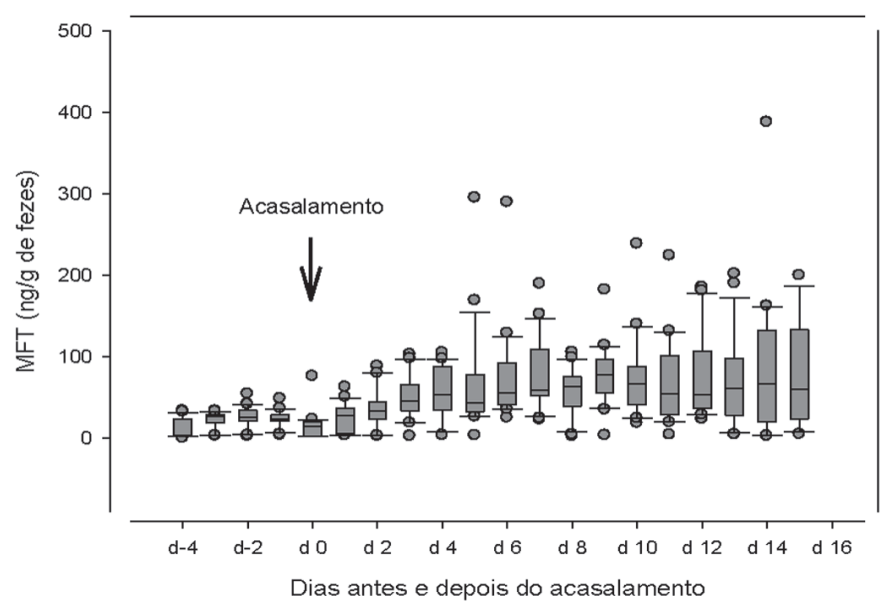

Fig.3. Concentrações de metabólitos fecais de testosterona (MFT) em 20 hamsters fêmeas prenhes antes e depois do acasalamento.

$t=0,5384,12 \mathrm{gl}, \mathrm{p}=0,60)$. Um aumento abrupto da concentração de MFT foi registrado no dia seguinte à administração do hormônio $(1811,4 \mathrm{ng} / \mathrm{g})$ chegando no segundo dia ao valor máximo de 1957,7ng/g. A concentração mantevese muito alta até o final das coletas quatro dias após a injeção. A injeção de solução salina não provocou nenhuma alteração nas concentrações de MFT do grupo contro-

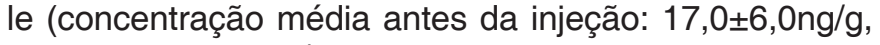
depois: $13,5 \pm 9,5 \mathrm{ng} / \mathrm{g}$; teste $t$ para amostras independentes $t=0,6759$, $7 \mathrm{gl}, \mathrm{p}=0,52$; Fig.2).

Entre as fêmeas gestantes, as concentrações de MFT variaram entre 8,3 e 33,2ng/g nos 6 dias que antecederam o acasalamento. A média das concentrações medidas nesses dias foi similar à concentração basal do grupo controle $(18,8 \pm 5,9 \mathrm{ng} / \mathrm{g}$; teste $t$ para amostras independentes, $t=1,594,133 \mathrm{gl}, p=0,1133)$. No entanto, a partir do segundo dia após o acasalamento, foi observado um aumento rápido, ainda que progressivo, destas concentrações. O teste $t$ para amostras pareadas confirmou a significância da diferença entre a concentração basal e as concentrações médias detectadas a partir do segundo dia de gestação até a véspera do parto. A concentração média máxima $(82,8 \pm 90,1 \mathrm{ng} / \mathrm{g})$ foi registrada no oitavo dia da gestação, porém a partir do quinto dia de gestação e até a véspera do parto, a concentração manteve-se em níveis similares ou até superiores ao nível basal dos machos (Fig.3).

\section{DISCUSSÃO}

O desafio com GnRH demonstrou claramente que a atividade gonadal, no hamster macho, é refletida nas concentrações fecais de metabólitos de testosterona. Da estimulação da síntese de testosterona resultou nas fezes um pico de MFT equivalente a $300 \%$ do nível basal. Seguiu-se uma queda de concentração abaixo do nível basal, sinal da inibição da síntese de testosterona pelo efeito de feedback negativo. As flutuações importantes das concentrações observadas nas fezes foram também relatadas no sangue, onde são comparáveis àquelas observadas no rato (Siegel 1985). O nível basal de MFT medido no presente estudo foi inferior ao nível basal medido nas fezes de cobaias (variação de 234,5 a 481,2ng/g; Bauer et al. 2008) ou de camundongos (173 $\pm 39 \mathrm{ng} /$ g; Billitti et al. 1998). Ademais, no camundongo, uma injeção de hCG elevou em quase $800 \%$ a concentração de MFT. Metodologias diferentes de mensuração talvez possam explicar parte dessas diferenças. O mais provável, no entanto é que elas estejam relacionadas com a menor capacidade individual de síntese de testosterona das células de Leydig do hamster quando comparado com outras espécies (Siegel 1985). Em conjunto, esses resultados confirmam a adequação do ensaio para o monitoramento da atividade do eixo hipotálamo-hipófise-gônadas (HPG) do hamster macho. Nossos resultados fornecem ainda informações novas e importantes sobre o prazo necessário para a metabolização e excreção da testosterona no macho: o sinal do pico de testosterona no sangue apareceu nas fezes produzidas entre oito e 12 horas mais tarde enquanto o pico negativo subseqüente era detectado nas fezes secretadas entre 16 e 20 horas após a administração de GnRH. Podemos estimar, portanto como de 8 a 12 horas a duração da metabolização e da excreção da testosterona no hamster macho. As oscilações das concentrações médias medidas nas 24 horas que antecederam a administração de hormônio confirmam esse resultado. De fato, foram medidas, no início do período claro concentrações mais altas do que no início do período escuro, o que sugere um ciclo circadiano de variação da concentração sanguínea de testosterona com acrofase 12 horas antes, ou seja, no início da noite. Este resultado está de pleno acordo com o ritmo circadiano da produção de testosterona relatado na literatura para o hamster macho (Vaughan et al. 1994). Na mesma espécie, prazos de 5 a 25 horas foram relatados para metabolização e excreção de esteróides ovarianos (Chelini et al. 2005) e de 20 horas para o aparecimento nas fezes dos machos do sinal da elevação dos níveis de glicocorticóides em resposta a um desafio por ACTH (Chelini et al. 2010).

Os resultados da administração de testosterona nas fêmeas, com um pico de MFT nas fezes excretadas nas 24 horas que seguiram a injeção sugerem que é também possível monitorar as alterações das concentrações sanguíneas deste hormônio usando radioimunoensaio. No entanto, a elevação acima do nível basal dos machos observada nas fêmeas gestantes está em contradição com dados da literatura segundo os quais a concentração sanguínea de testosterona se mantém baixa ao longo da gestação (Soares \& Talamantes 1982). Baseada na dose administrada diariamente a hamsters machos por Huppenbauer et al. (2005), a quantidade de hormônio injetada nas fêmeas parece, porém ter elevado a concentração sanguínea muito acima do nível fisiológico. É possível, então que a capacidade das vias normais de metabolização da testosterona nas fêmeas tenha sido ultrapassada, o que pode ter resultado na excreção de metabólitos diferentes ou mesmo do próprio hormônio, causando a elevação medida pelo RIE. As concentrações relativamente altas de MFT medidas nas fêmeas gestantes comparadas com os baixos níveis séricos descritos na literatura sugerem a ligação ao 
anticorpo de outras moléculas, talvez andrógenos de origem adrenal, além de metabólitos de testosterona. Situações semelhantes foram descritas na cobaia, com ausência de correlação entre concentrações séricas de testosterona e concentrações de MFT medidas por enzimaimunoensaio (Bauer et al. 2008), e em diversas espécies de primatas (Möhle et al. 2002). Uma análise bioquímica precisa, por cromatografia gasosa acoplada a espectrometria de massa, por exemplo, seria necessária para determinar a natureza dos principais metabólitos de testosterona presentes nas fezes da fêmea de hamster e permitir o desenvolvimento de um ensaio adequado.

Em conclusão, o presente estudo demonstra pela primeira vez a adequação de um conjunto diagnóstico comercial para a quantificação dos metabólitos fecais de testosterona e a investigação da atividade do eixo HPG no hamster Sírio macho. Trata-se de uma ferramenta não invasiva de grande utilidade para futuros estudos envolvendo tanto a função reprodutivo quanto o comportamento social deste animal. Esta alternativa ao uso de amostras de sangue descortina a possibilidade de estudos de longo prazo com um número mínimo de animais, garantindo o seu bem-estar. O mesmo ensaio, entretanto, parece ter medido, nas fêmeas, metabólitos de outros esteróides, resultado que confirma, uma vez mais, a necessidade de validar cuidadosamente as técnicas de dosagem de esteróides fecais não somente para cada espécie como para cada sexo de uma mesma espécie.

Agradecimentos.- À Técnica de Laboratório Priscila Viau Furtado pela realização dos ensaios. À Fundação de Amparo à Pesquisa do Estado de São Paulo pela concessão de bolsa de doutorado e de auxílio à pesquisa; e ao Conselho Nacional de Desenvolvimento Científico e Tecnológico (CNPq).

\section{REFERÊNCIAS}

Bajusz E. 1969. Hereditary cardiomyopathy: A new disease model. Am. Heart J. 77:686-696.

Bauer B., Palme R., Machatschke I.H., Dittami J. \& Huber S. 2008. Non-invasive measurement of adrenocortical and gonadal activity in male and female guinea pigs (Cavia aperea f. porcellus). Gen. Comp. Endocrinol. 156:482-489.

Billitti J.E., Lasley B.L. \& Wilson B.W. 1998. Development and validation of a fecal testosterone biomarker in Mus musculus and Peromyscus maniculatus. Biol. Reprod. 59:1023-1028.

Cain S.W., Karatsoreos I., Gautam N., Konar Y., Funk D., McDonald R.J. \& Ralph M.R. 2004. Blunted cortisol rhythm is associated with learning impairment in aged hamster. Physiol. Behav. 82:339344.

Chelini M.O.M., Souza N.L., Rocha A.M., Felippe E.C.G. \& Oliveira C.A. 2005. Quantification of fecal estradiol and progesterone metabolites in Syrian hamster (Mesocricetus auratus). Braz. J. Med. Biol. Res. 38: 1711-1717.

Chelini M.O.M., Souza N.L., Cortopassi S.R.G., Felippe E.C.G. \& Oliveira C.A. 2006. Assessment of the physiologic stress response by quantification of fecal corticosteroids, J. Am. Assoc. Lab. Anim. 53:8-11.

Chelini M.O.M., Otta E., Yamakita C. \& Palme R. 2010. Sex differences in the excretion of fecal glucocorticoid metabolites in the Syrian hamster (Mesocricetus auratus). J. Comp. Physiol. B180(6):919-25.
Cordner A.P., Herwood M.B., Helmreich D.L. \& Parfitt D.B. 2004. Antidepressants blunt the effects of inescapable stress on male mating behaviour and decrease corticotropin-releasing hormone mRNA expression in the hypothalamic paraventricular nucleus of the Syrian hamster (Mesocricetus auratus). J. Neuroendocrinol. 16:628-636.

Dale P.P., Lazansky J. \& Keyes P.H. 1944. Production and inhibition of dental caries in Syrian hamsters, preliminary observations. J. Dent. Res. 23:445-451.

Dias E.A., Nichi M. \& Guimarães M.A.B.V. 2008. Comparison of two commercial kits and two extraction methods for fecal glucocorticoid analysis in ocelots (Leopardus pardalis) submitted to ACTH challenge. Pesq. Vet. Bras. 28:329-334.

Gattermann R., Fritzsche P., Neumann K., Al-Hussein I., Kayser A., Abiad M. \& Yakti R. 2001. Notes on the current distribution and the ecology of wild golden hamsters (Mesocricetus auratus). J. Zool., Lond., 254:359-365.

Gebhardt-Heinrich S.G., Fischer K., Hausenberger A.R., Keller P. \& Steiger A. 2007. The duration of capture and restraint during anesthesia and euthanasia influences glucocorticoid levels in male golden hamsters. Lab. Anim. 36:41-46.

Huppenbauer C.B., Tanzer L., DonCarlos L.L. \& Jones K.J. 2005. Gonadal steroid attenuation of developing hamster facial motoneuron loss by axotomy: Equal efficacy of testosterone, dihydrotestosterone, and 17- $\beta$ estradiol. J. Neurosci. 25:4004-4013.

Lepschy M., Touma C., Hruby R. \& Palme R. 2007. Non-invasive measurement of adrenocortical activity in male and female rats. Lab. Anim. 41:372-387.

Missihoun C., Zisa D., Shabbir A., Lin A. \& Lee T. 2009. Myocardial oxidative stress, osteogenic phenotype, and energy metabolism are differentially involved in the initiation and early progression of $\delta$-sarcoglycan-null cardiomyopathy. Mol. Cell Biochem. 321:4552.

Möhle U., Heistermann M., Palme R. \& Hodges J.K. 2002. Characterization of urinary and fecal metabolites of testosterone and their measurement for assessing gonadal endocrine function in male nonhuman primates. Gen. Comp. Endocrinol. 129:135-145.

Siegel H. 1985. The Hamster. Reproduction and Behavior. Plenum Press, New York. 440p.

Soares M.J. \& Talamantes F. 1982. Placental and serum hormone changes during the second half of pregnancy in the hamster. Biol. Reprod. 27:523-529.

Taravosh-Lahn K. \& Delville Y. 2004. Aggressive behavior in female golden hamsters: Development and the effect of repeated social stress. Horm. Behav. 46:428-435.

Touma C., Fenzl T., Ruschel J., Palme R., Holsboer F., Kimura M. \& Landgraf R. 2009. Rhythmicity in mice selected for extremes in stress reactivity: Behavioural, endocrine and sleep changes resembling endophenotypes of major depression. PLoS ONE 4(1):e4325.

Vaughan M.K., Menendez-Pelaez A., Buzzell G.R., Vaughan G.M., Little J.C. \& Reiter R.J. 1994. Circadian rhythms in reproductive and thyroid hormones in gonadally regressed male hamsters exposed to natural autumn photoperiod and temperature conditions. Neuroendocrinology 60:96-104.

Wommack J.C., Salinas A., Melloni Jr R.H. \& Delville Y. 2004. Behavioural and neuroendocrine adaptations to repeated stress during puberty in male golden hamsters. J. Neuroendocrinol. 16:767-775.

Zhang J.Z., Rao X.P., Sun L., Wang D.W., Liu D. \& Zhao C. 2008. Cohabitation impaired physiology, fitness and sex-related chemosignals in golden hamsters. Physiol. Behav. 93:1071-1077.

Zheng Sun, Sandeep Sood, Ning Li, Peiying Yang, Newman R.A., Chung S.Yang \& Xiaoxin Chen. 2008. Chemoprevention of 7, 12dimethylbenz[a]anthracene (DMBA)-induced oral carcinogenesis in hamster cheek pouch by topical application of a dual inhibitor of epidermal growth factor receptor (EGFR) and ErbB2 tyrosine kinases. Oral Oncol. 44:652-657. 